\title{
Current Trends in Veteran Suicides - From 2001 to 2019
}

\author{
Authors \\ Avinash Sharma, MSE ${ }^{1}$ \\ ${ }^{1}$ SoKat Consulting, LLC (www.sokat.com)
}

\begin{abstract}
In 2019, Veterans made up 8\% of the total US adult population (19.8 million vs 255 million). However, the percentage of Veteran suicide was $14 \%$ of US adult suicides $(6,261$ vs 45,861$)$. The US Department of Veterans Affairs (VA) annually releases data on Veterans' suicide, categorized by gender, age group, geographical region, and states. The 2021 National Veteran Suicide Prevention Annual ${ }^{1}$, released in September 2021, provides a concrete summary of the issues related to Veteran suicide and the VA's approach to solving them. This article builds on that report and provides additional perspectives on these suicide numbers, using features such as age group and geographical regions. This work aims to get a better understanding and insights into the Veterans' suicide data. All analysis was conducted using the data published by the US Department of Veterans Affairs (VA) Office of Mental Health and Suicide Prevention ${ }^{1}$. Two datasets were used: 1) National Veteran Suicide Data and Reporting - Data Appendix (2019), and 2) State-Level Veteran Suicide Data: 2019 Update - State Data Appendix.
\end{abstract}

\section{Total Veteran Suicides}

In 2019 alone, 6,261 Veterans died by suicide in the US, versus 39,600 deaths amongst the nonVeterans. While still considerably high, there has been a steady decline in the number of Veteran suicides in the last two years. Total Veteran deaths by suicide have been at their lowest point since 2008. Since 2001, the Veteran population has also consistently been reducing. In 2019, there were a total of 19.8M Veterans in the US, approximately $2 \%$ lower than the previous year (2018). It is therefore essential to consider the suicides rate instead of the total magnitude.

\footnotetext{
1 "VA.gov: Veterans Affairs." Veteran Suicide Data and Reporting. September 10, 2021. Accessed September 16, 2021. https://www.mentalhealth.va.gov/suicide_prevention/data.asp.
} 


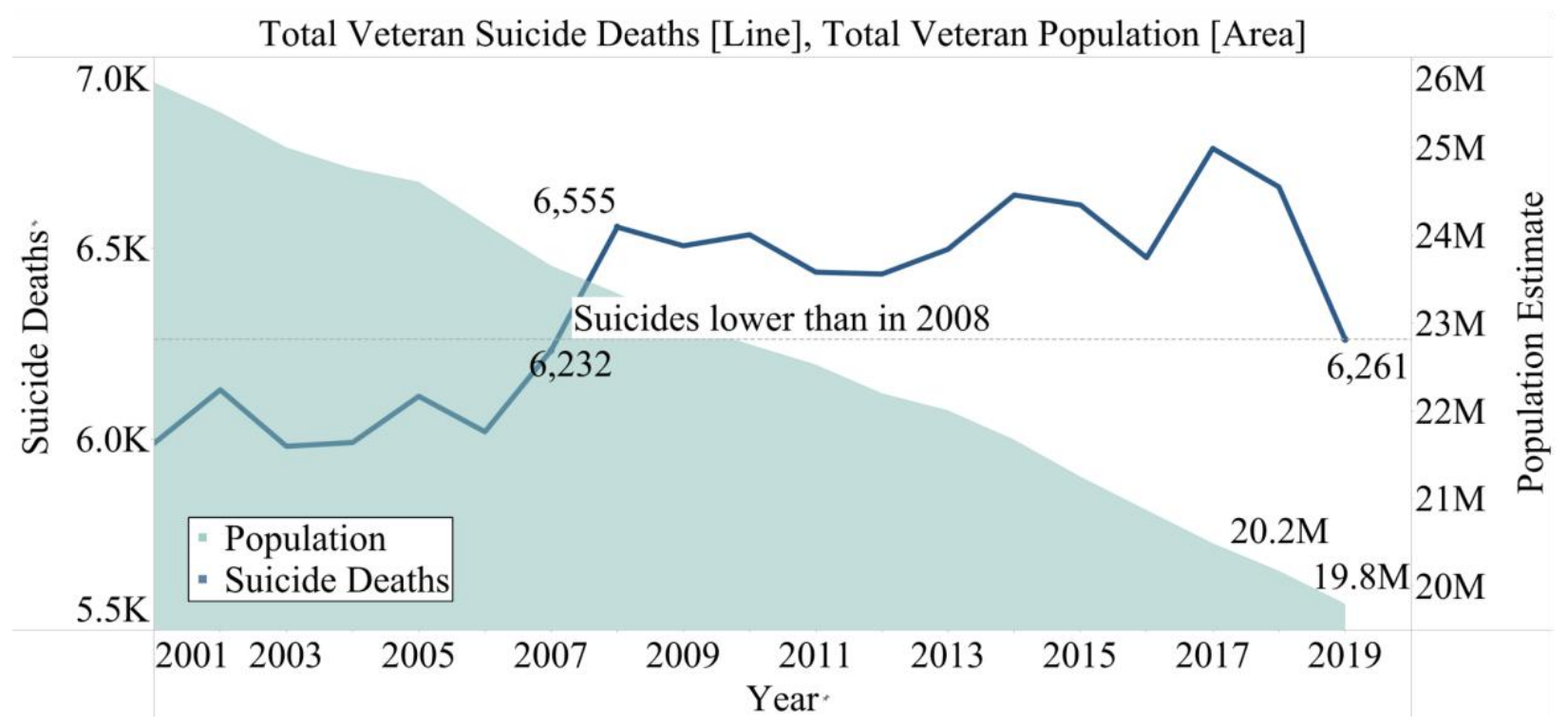

\section{Veteran Suicide Rate}

The Veteran suicide rate, when adjusted for age and gender, was at $26.9 *$ in 2019 . This rate has consistently seen a decrease in the last two years. In 2017, the Veteran suicide rate was 29.4*, the highest since 2001. The lowest was $16.8 *$ in 2002.

\section{Veteran Suicide Rates (age and gender adjusted)}

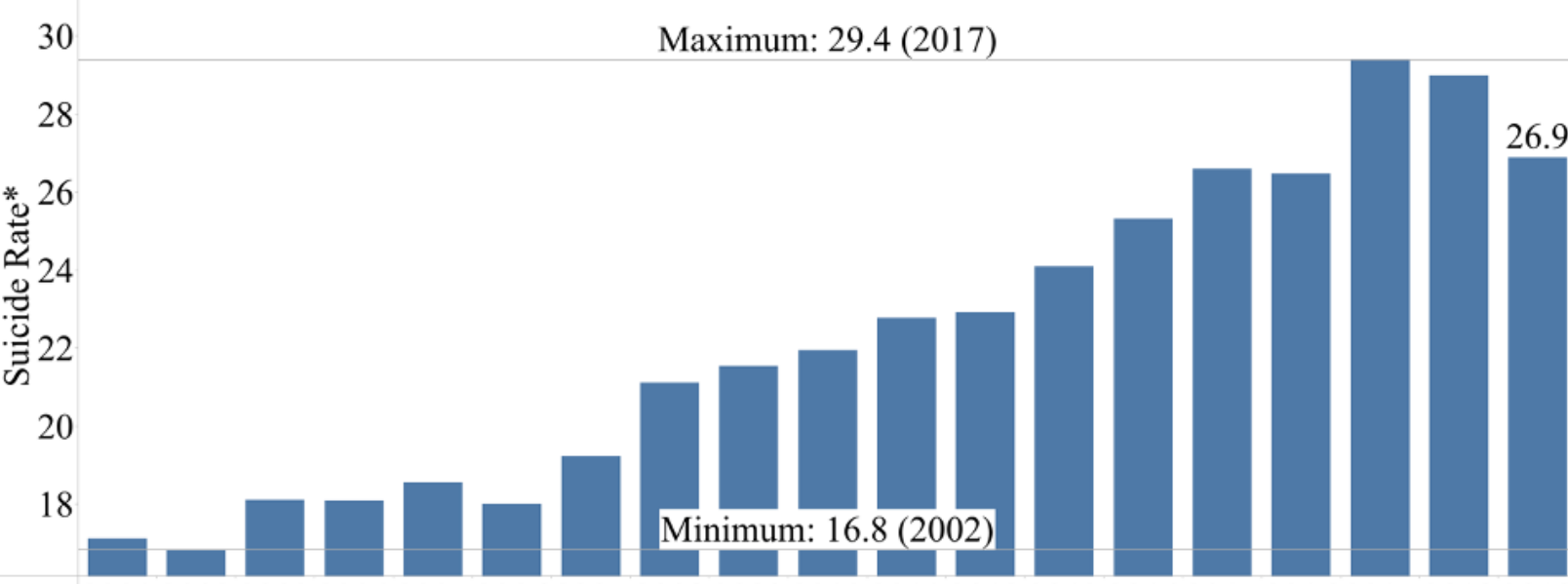

2001200220032004200520062007200820092010201120122013201420152016201720182019

* Suicide Rate per 100,000 of population

\section{Across Genders}

The male to female Veteran Population ratio was approximately 9:1 in 2019. In 2018, 9.7\% of the total Veteran population was female, which increased to $10.1 \%$ in 2019 - a $3 \%$ increase. In terms of suicide rates, both genders have seen a reduction in 2019. Male suicide rates are still more than double $(2.5 \mathrm{x})$ than that of female Veterans. In 2019 , the male suicide rate was $38.8^{*}$, the lowest 
since 2017. The female suicide rate in 2019 was $15.4^{*}$, close to the rate in 2016 . Both of these values are adjusted for age.

\section{Veteran Suicide Rates (age-adjusted) by Gender}

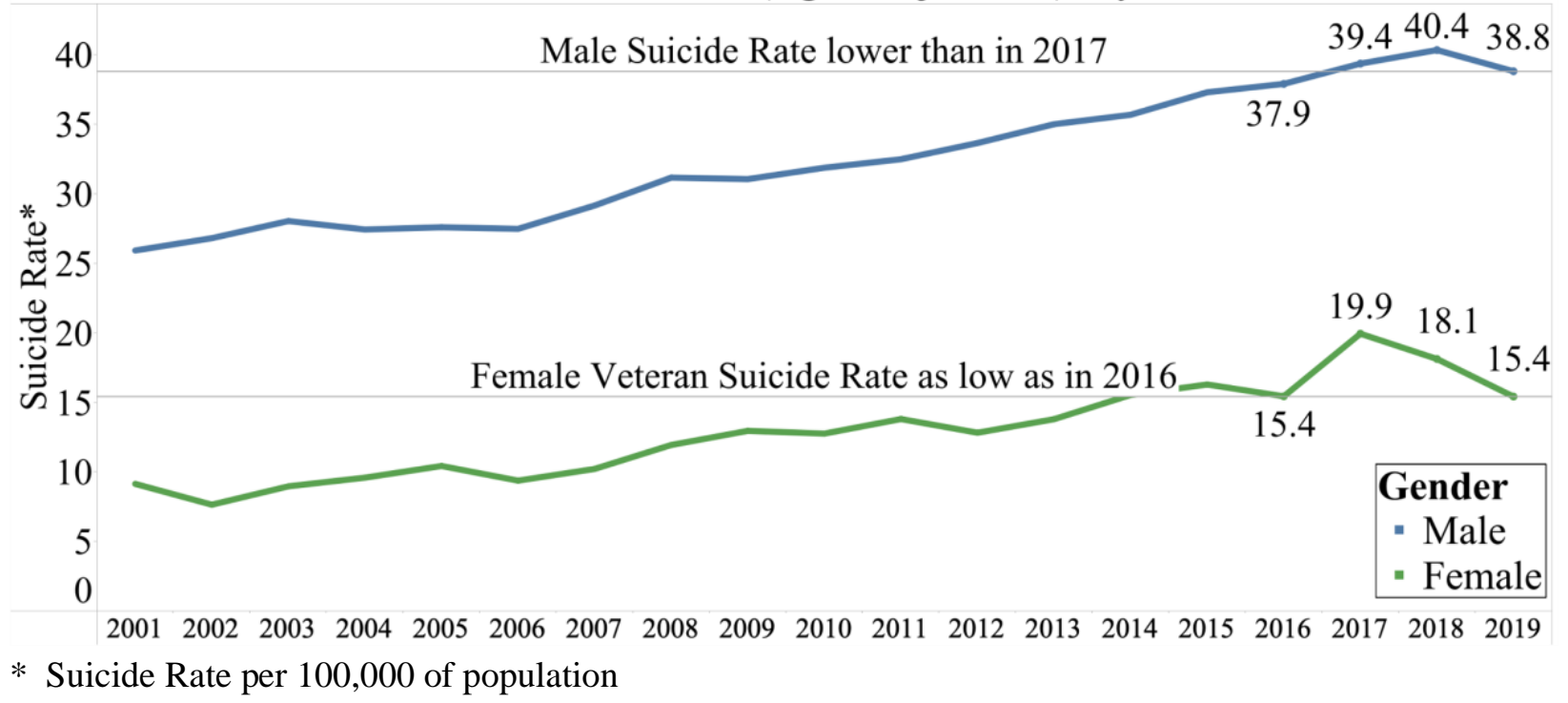

Given the suicide rate for every year, we looked at the percentage change in these rates over the years. For example, for male Veterans, we calculated the percentage change between $40.4 *$ (2018) and 38.8* (2019). We defined this as the year-over-year (YoY) growth or change in the suicide rate.

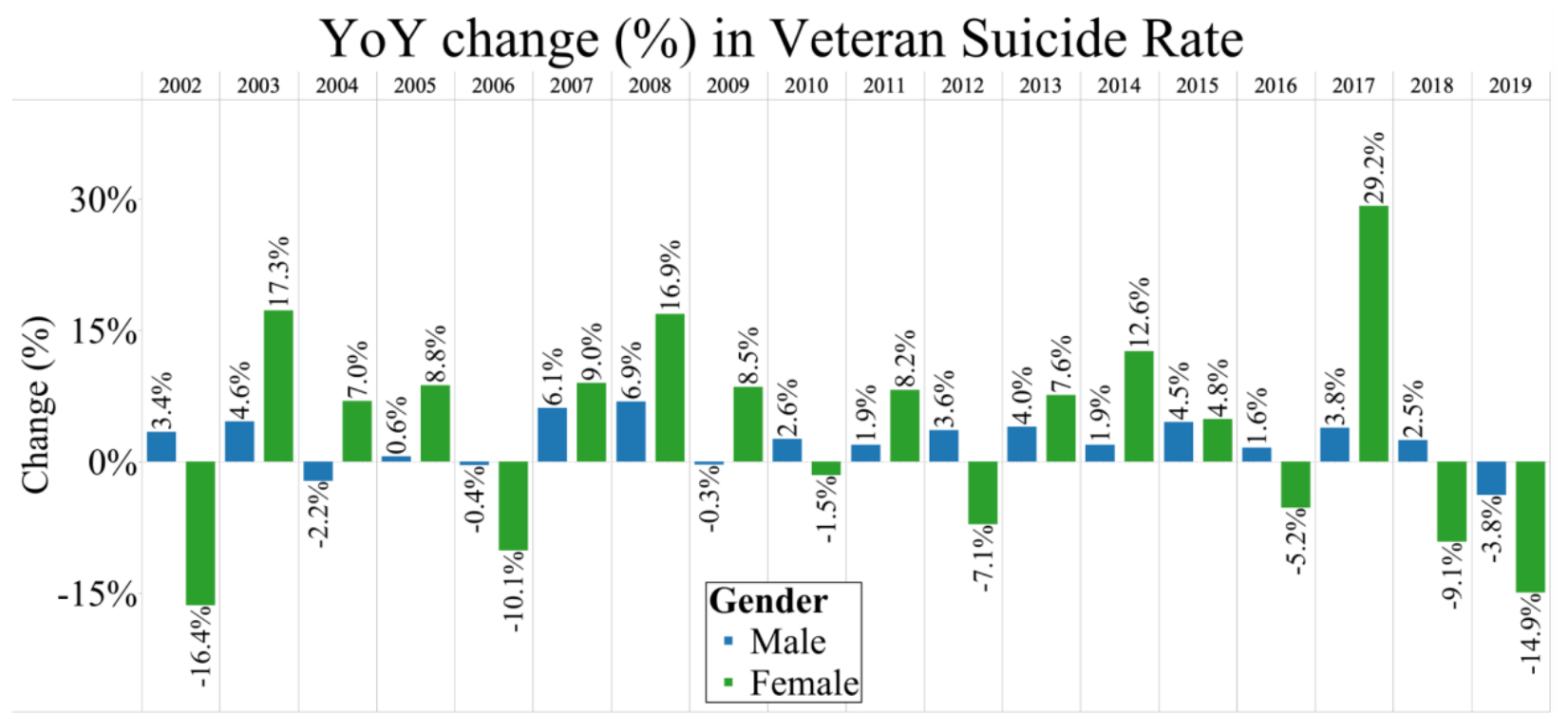


The following formula below was used to calculate this year-over-year (YoY) change:

$$
\begin{gathered}
\text { YoY change in Veteran Suicide Rate }(\%) \\
=100 \times \frac{\text { Veteran Suicide Rate }(\%)_{Y 1}-\text { Veteran Suicide Rate }(\%)_{Y 0}}{\text { Veteran Suicide Rate }(\%)_{Y 0}}
\end{gathered}
$$

Y1: current year, Y0: previous year

The above metric provides us with a rate at which the Veteran suicide rate changed from one year from its previous year. Between 2018 (YO) and 2019 (Y1), the male suicide rate decreased by $3.8 \%$, while for females, it decreased by $14.8 \%$. The graph above illustrates the YoY change in Veteran Suicide Rate between 2002 and 2019 for both genders. Note that since we calculate the change over the previous year, 2001 is excluded from the graph.

\section{Across Age Groups}

2019 has seen a decrease in suicide rates in all age groups. There was an $8.3 \%$ reduction in suicide rates in the youngest group, 18-34. This group also saw the highest percentage reduction in suicide rates in 2019. Before that, 2017 and 2018 had the highest suicide rates since 2001.

Year with highest suicide rate across age groups:

18-34: $2018\left(48^{*}\right)$

35-54: $2017\left(34^{*}\right)$

55-74: $2018\left(31^{*}\right)$

$75+: 2017\left(33^{*}\right)$

* Suicide Rate per 100,000 of population

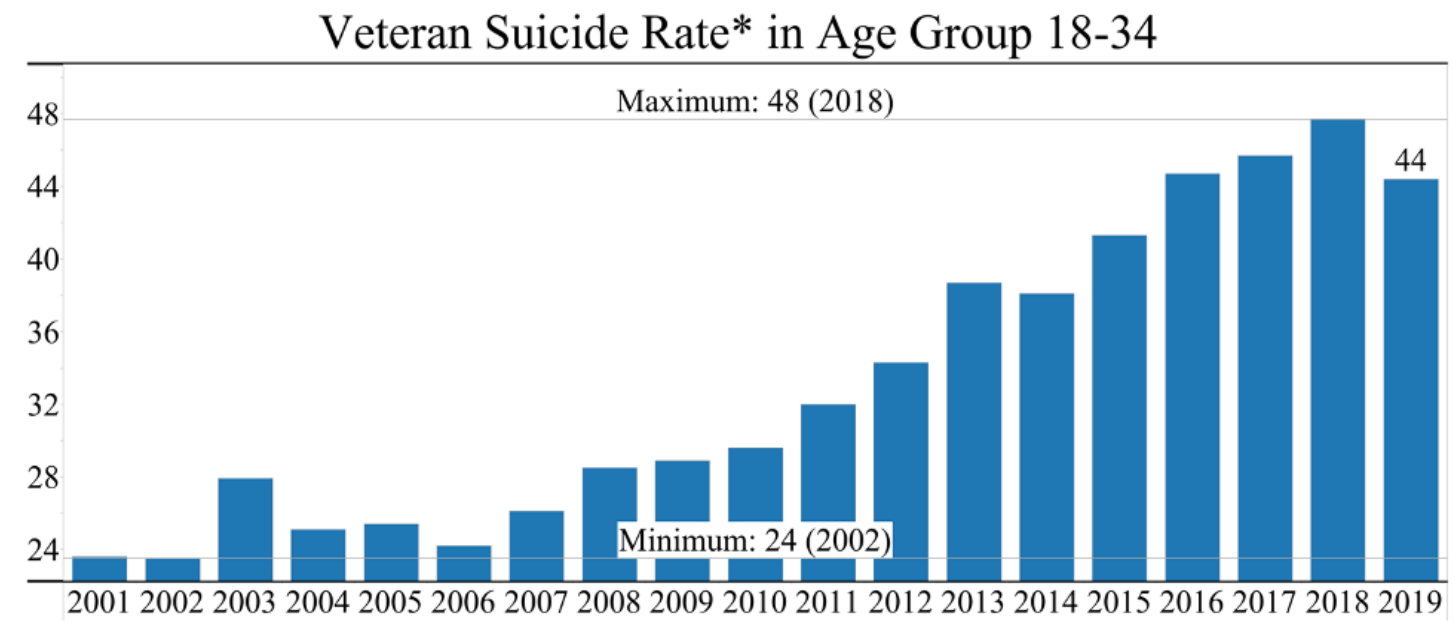



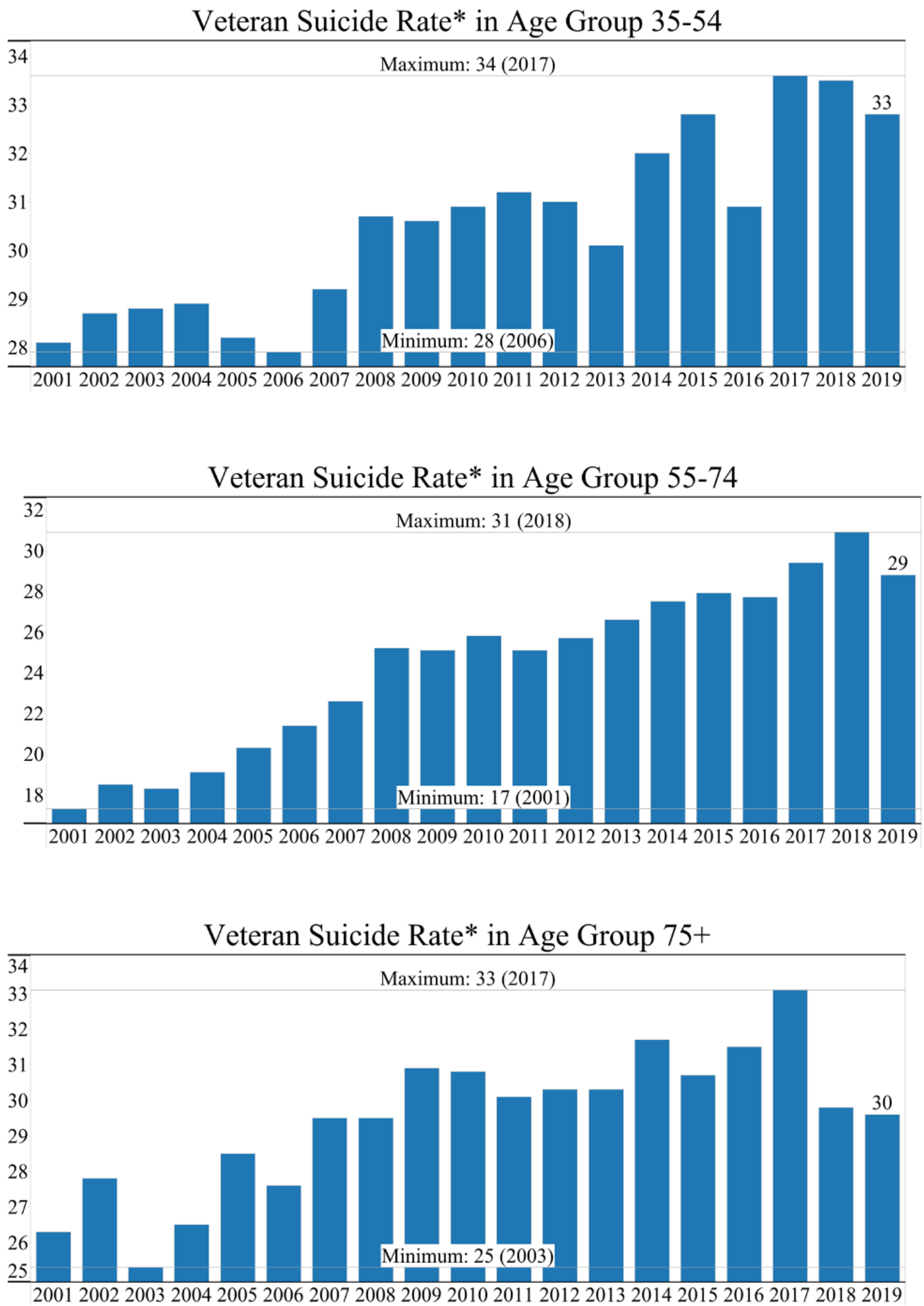

* Suicide Rate per 100,000 of population 


\section{By Geographic Region}

Veteran Suicide Rates were analyzed by each state and geographic region. These geographic regions include Midwest, Northeast, South, and West. At a state level, we observed the change in the Veteran suicide rate between 2019 and 2018. This is calculated as follows:

$$
\begin{gathered}
\text { Veteran Suicide Rate change }(\%){ }^{\#} \\
\text { Veteran Suicide Rate }(\%)_{2019}-\text { Veteran Suicide Rate }(\%)_{2018} \\
\text { Veteran Suicide Rate }(\%)_{2018}
\end{gathered}
$$

${ }^{\#}$ Lower is better

Note that this same as the YoY change in Veteran Suicide (\%) we calculated year, with $Y 1=2019$, and $Y O=2018$. As some states had low suicide deaths, their suicide rates were unreliable for analysis. After excluding such states, we looked at 44 states and calculated their change in Veteran suicide rate based on the above formula. The map below provides a visualization of this. States such as Maryland (-29\%), Alabama (-25\%), and Maine (-23\%), have seen a significant decrease in Veteran suicide rates. In comparison, states such as Nebraska (22\%), Nevada (18\%), and Iowa $(17 \%)$ have seen the highest increase.

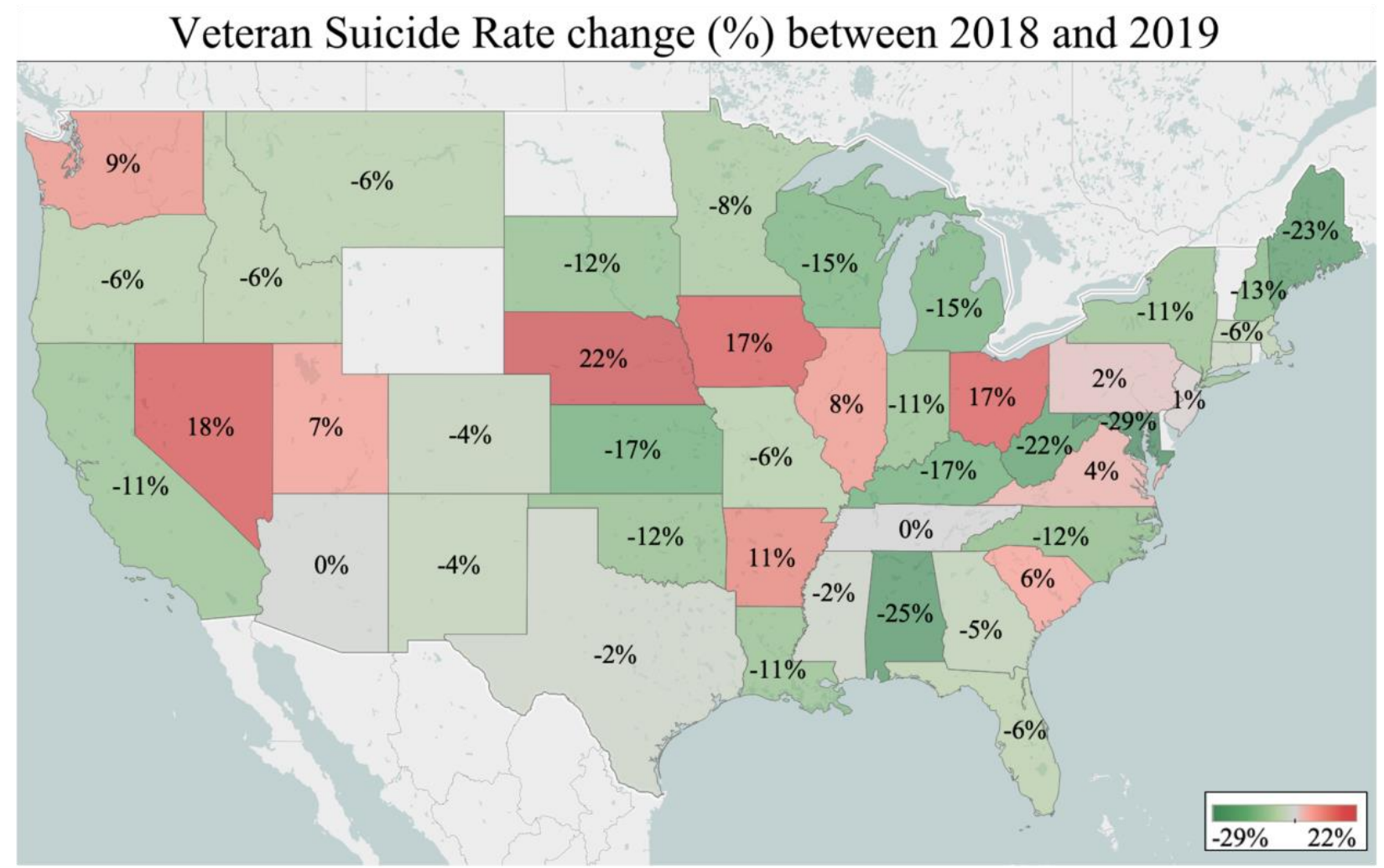


Age Group \& Region:

In this section, we add age groups to the geographic regions component and perform similar analyses. We show (for each age group) the geographic regions and age groups that saw an increase in the suicide rate in 2019. The age groups 55-74 and 75+ both saw a decline in the suicide rates across the four regions. They have therefore not been included here. Their charts can, however, be found in the appendix section.

Age Group 18-34:

In 2019, there was an increase in the suicide rates for this group in the West and Northeast regions. Both these regions also have the highest suicide rates since 2001 in this age group.
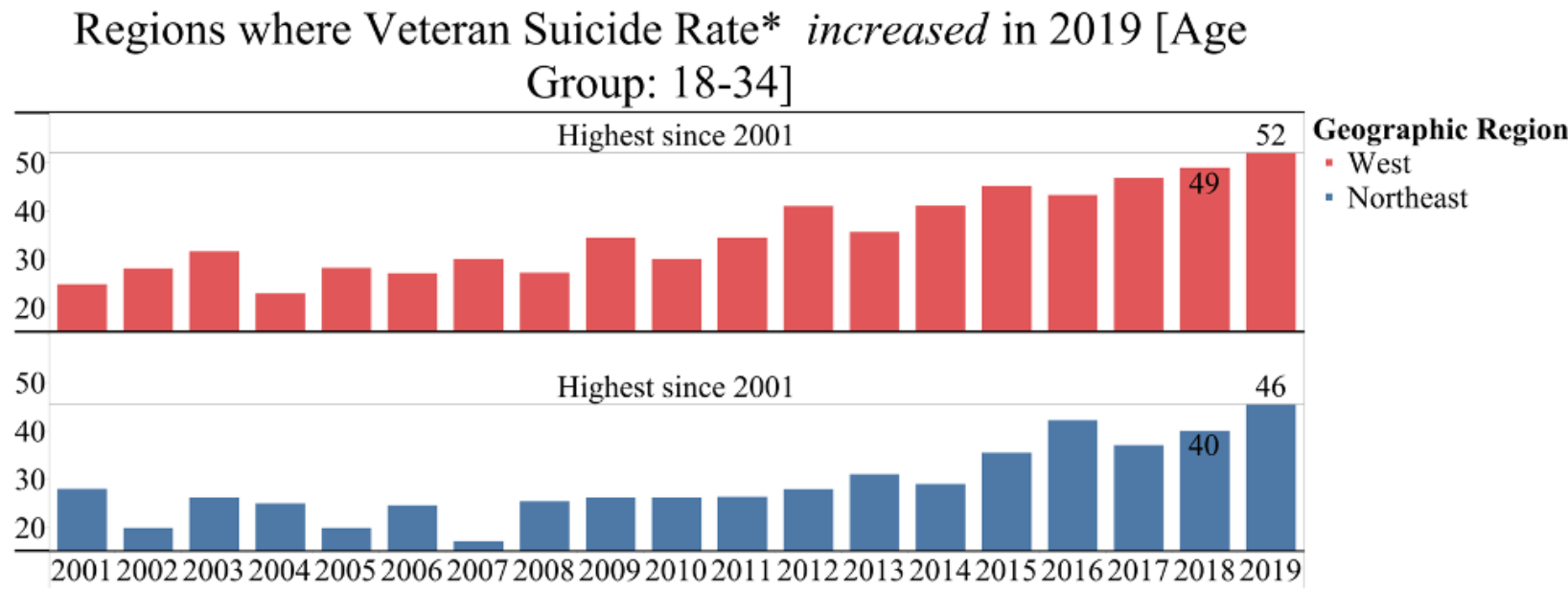

\section{Age Group 35-54:}

In 2019, there was an increase in the suicide rates for this group in the Midwest and Northeast regions. Both these regions also have the highest suicide rates since 2001 in this age group.

\section{Regions where Veteran Suicide Rate* increased in 2019 [Age}

Group: $35-54]$

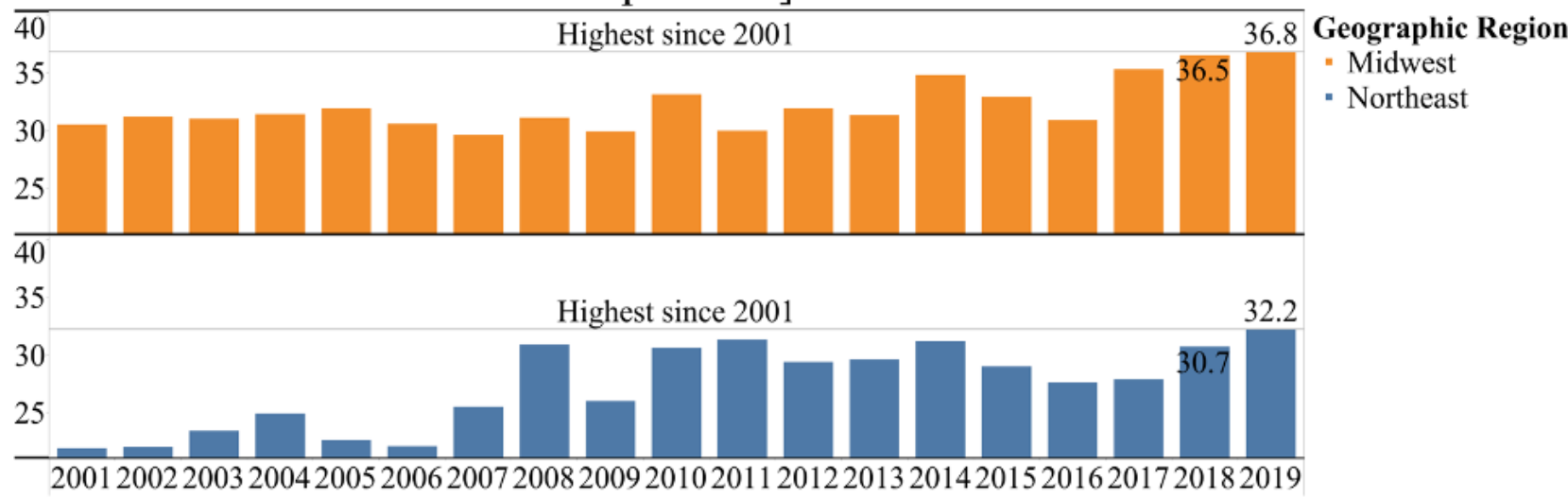

* Suicide Rate per 100,000 of population 


\section{Age Group \& States}

For each state, we pulled out the age groups with the highest suicide rate in 2019. The map below provides a visual on this. 7 states (including the District of Columbia) did not provide this data. Those have, therefore, not been included in the map. Amongst the 44 states that provided this data, 22 states had the youngest age group, 18-34, with the highest suicide rates. Alaska is the only state which had the age group $75+$ with the highest suicide rate.

\section{Age Groups with Highest Veteran Suicide Rate* across states}

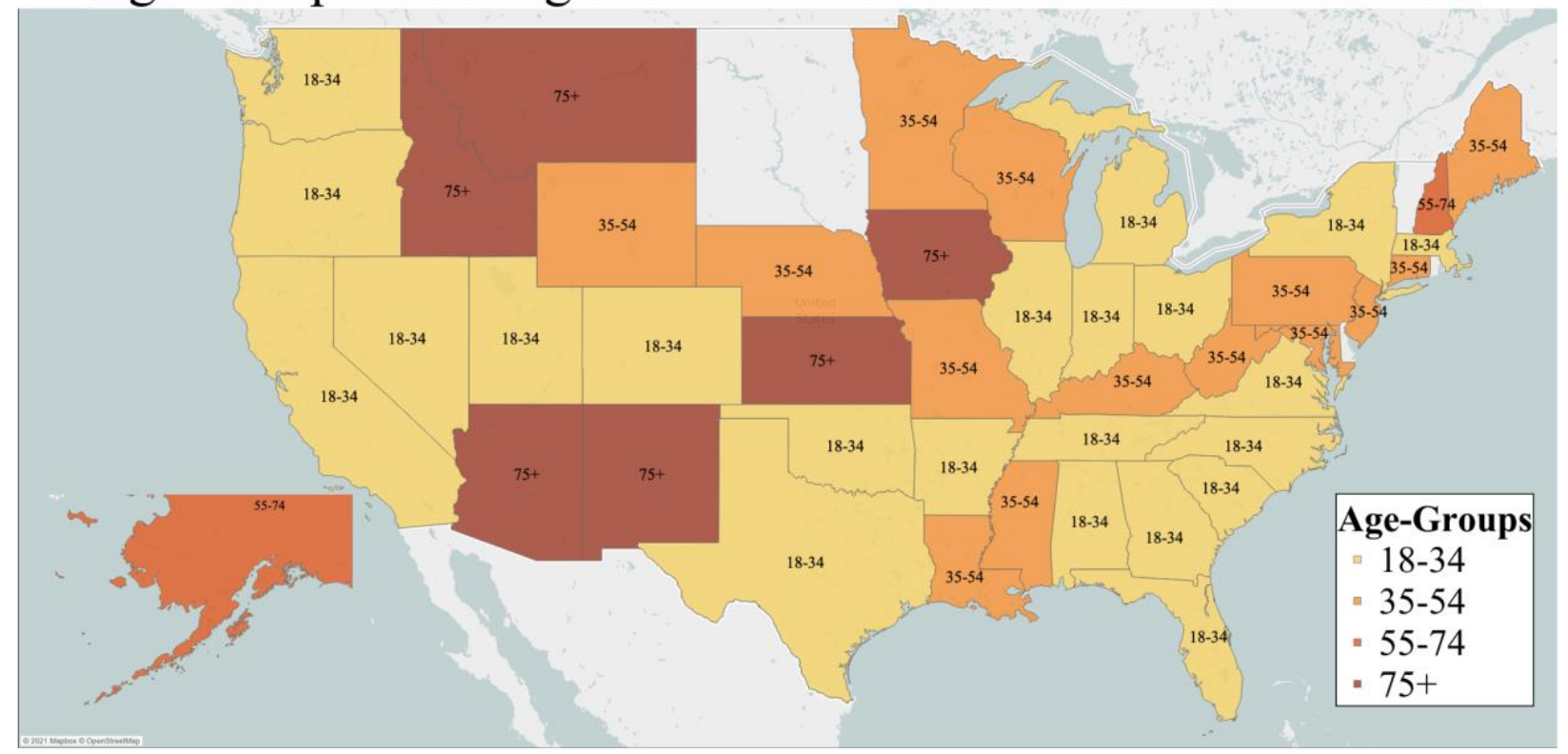

\section{Method of Suicide}

Since 2001, firearms have always had the highest contribution to suicide methods amongst Veterans. This trend remained the same in 2019, with firearms contributing to $69 \%$ of Veterans' total suicides. This was followed by suffocation (17\%), poisoning (8\%), and other suicide methods (5\%). In 2019, Veteran suicide by Firearm constituted more than $80 \%$ (8 in 10) deaths in at least 5 US states. Mississippi is one of the states with the highest Firearm contribution to Veteran suicides, followed by Louisiana, Tennessee, Indiana, and Alabama. 
Veteran Suicide Method

(\%) in 2019

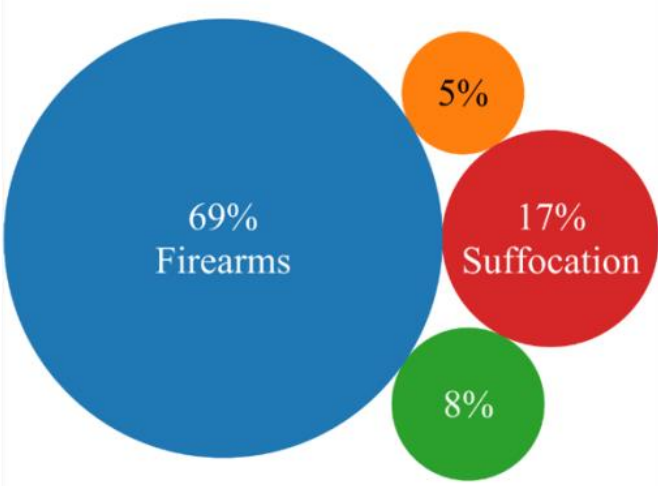

States with highest Veteran Suicide by Firearms in 2019

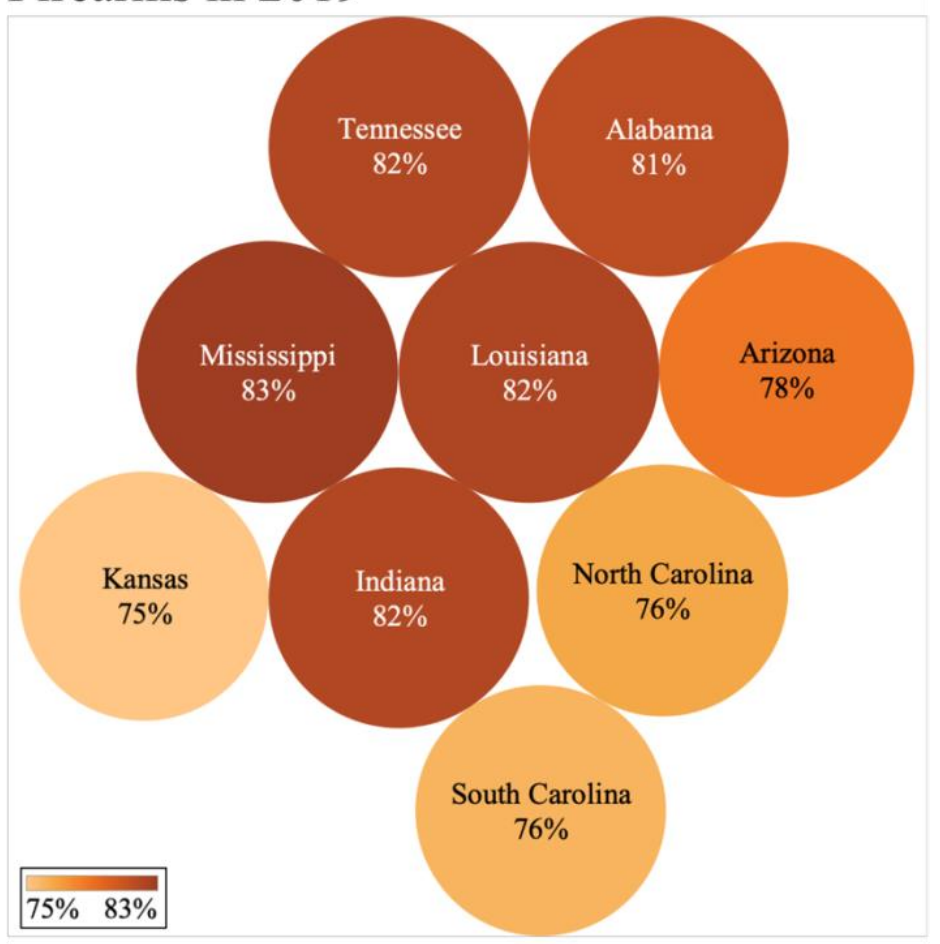

In certain states, there has been an increased contribution to Veterans' suicide by firearms. The map below shows the top 10 states that have seen an increase in suicide by firearms between 2018 and 2019. Nebraska and Indiana have seen some of the highest increases of $27 \%$ and $26 \%$, respectively. In Nebraska, the contribution percentage of suicide through Firearms increased from $52.4 \%$ in 2018 to $66.7 \%$ in 2019. Indiana had a similar increase from $68.8 \%$ in 2018 to $81.8 \%$ in 2019. These changes were calculated using the following formula:

Change $(\%)$
$=100 \times \frac{\text { Percentage Suicide by Firearms }(\%)_{2019}-\text { Percentage Suicide by Firearms }(\%)_{2018}}{\text { Percentage Suicide by Firearms }(\%)_{2018}}$ 
Top 10 states with an increase in Veteran Suicide contribution by Firearms (\%) between 2018 and 2019

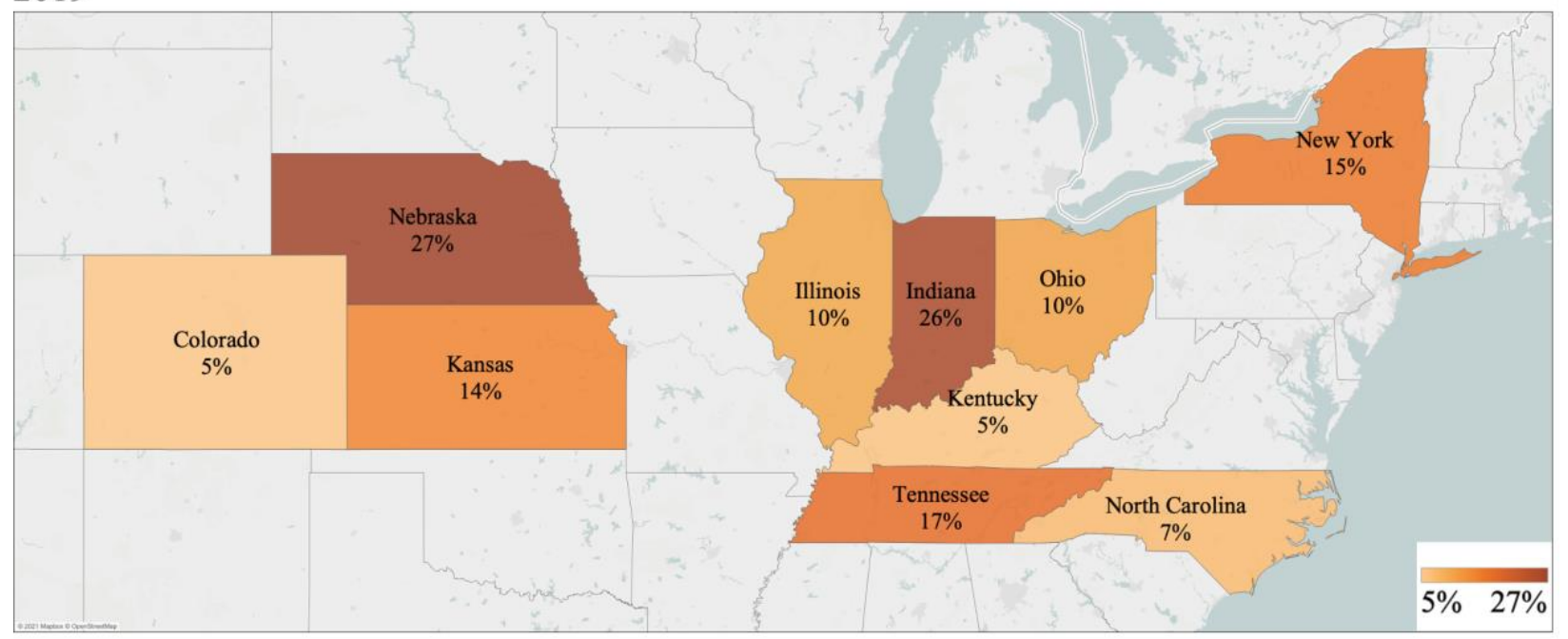

\section{Discussion}

The VA Office of Mental Health and Suicide Prevention released nine years (2001-2019) of Veteran suicide data that this article dived into. While the raw Veteran suicide numbers are still high (6,261), 2019 has seen some promising improvements in several aspects. Overall, the Veteran suicide rate, after being normalized for age and gender, has seen a reduction, second year in a row.

Both genders have seen a drop in suicide rates in 2019. For male Veterans, the YoY change in the suicide rate between 2018 and 2019 was $-3.8 \%$. This is one of the most considerable improvements we have seen in recent years. The highest decrease at this rate for male Veterans was in 2004, where the drop was $-2.2 \% .2010$ and 2011 had a drop of $-0.3 \%$ and $-1.5 \%$, respectively. All other years saw an increase in male suicide rates. With that in mind, $-3.8 \%$ in 2019 was encouraging. Similarly, the change in the suicide rate for female Veterans was $-14.9 \%$ between 2018 and 2019, the highest reduction we have seen since 2002.

At the country level, we observed a reduction in the suicide rate across all age groups in 2019 . The youngest age group, between 18-34, still has the highest suicide rate in 2019. In at least 22 states, Veterans in this age group have suicide rates higher than other age groups. At the state level, at least 31 states saw a decrease in the Veteran suicide rate. In states like Maryland, Alabama, Maine, and West Virginia suicide rate reduced by more than $20 \%$ between 2018 and 2019. On the contrary, states like Nebraska, Nevada, and Iowa, had some of the largest increases in suicide rates in the same period.

In some regions and age groups, we saw an increase in the suicide rate. In the youngest group, ages 18 to 34, the West and Northeast regions saw increased suicide rates and the highest since 2001. In the most populous age group, ages 35 to 54, the Midwest and Northeast saw increased 
suicide rates and are at their highest since 2001. These areas need extra attention in Veteran mental health management.

Firearms have been the most common method of suicide amongst Veterans since 2001. Approximately 7 in 10 Veteran suicides are with firearms. States such as Mississippi, Louisiana, Indiana, Tennessee, and Alabama, have more than $80 \%$ of Veteran suicides by firearm. The suicide death rate by firearm has increased in states like Nebraska, Indiana, Tennessee, and New York between 2018 and 2019.

This article has highlighted some of the recent developments in Veteran suicide statistics. There are still questions that need further investigation. Some of the key ones include analyzing and deriving the factors that have led to these changes. The VA has seen an increase in its spending by introducing several new initiatives such as the Suicide Prevention 2.0 initiative (SP 2.0), Universal Screening, or expansion of the MyVA access. It would be interesting to see the effects of these initiatives on the Veteran suicide rates. Another aspect would be to include the nonVeteran population in the above analyses. Comparing the Veteran population with the nonVeteran population and observing differences and correlations in these trends will also help us gain additional insights. While we hope that the current trend of reduction in Veteran suicide continues, the COVID-19 pandemic may offset some of it. In January 2020, the US reported its first case of the virus. Since then, approximately 14,000 Veterans have succumbed to this virus. With record unemployment and increased mental stress, the pandemic has had unprecedented effects on the socio-economic conditions of Americans. It would be imperative to see how this affects suicide rates amongst Veterans in 2020. 


\section{Appendix}

Veteran Suicide Rate* in Age Group 18-34 by region

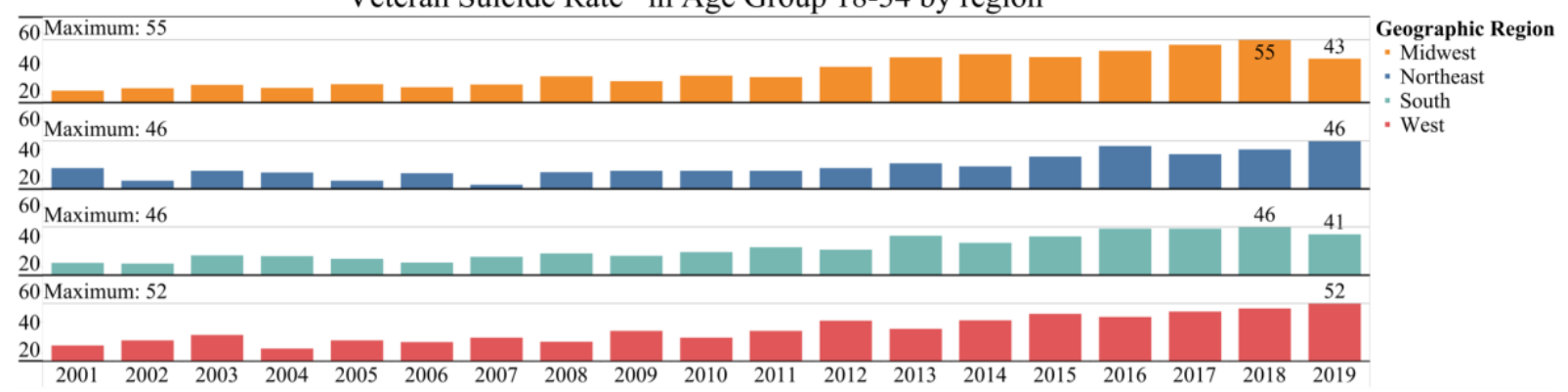

Veteran Suicide Rate* in Age Group 35-54 by region

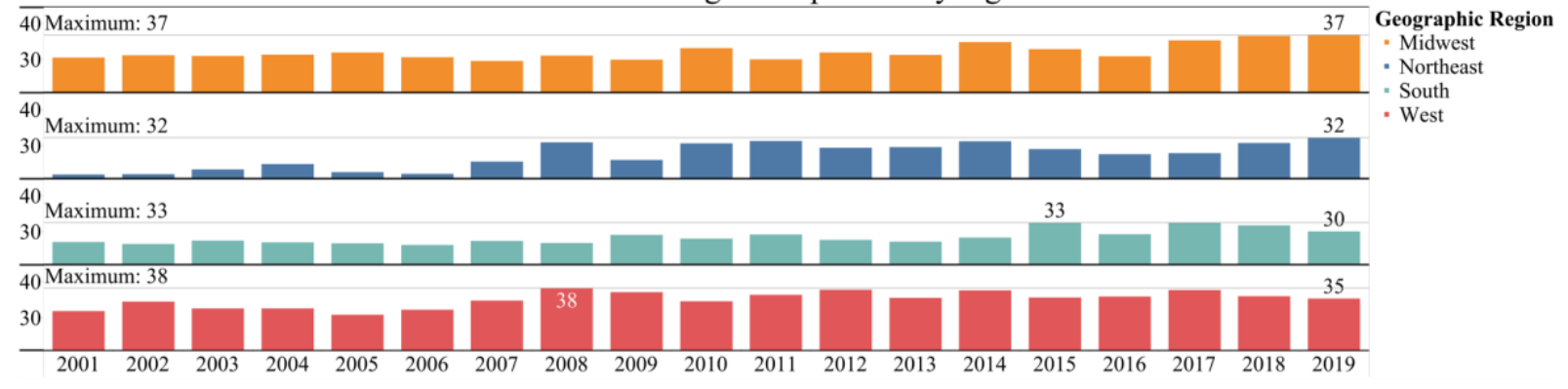

Veteran Suicide Rate* in Age Group 55-74 by region

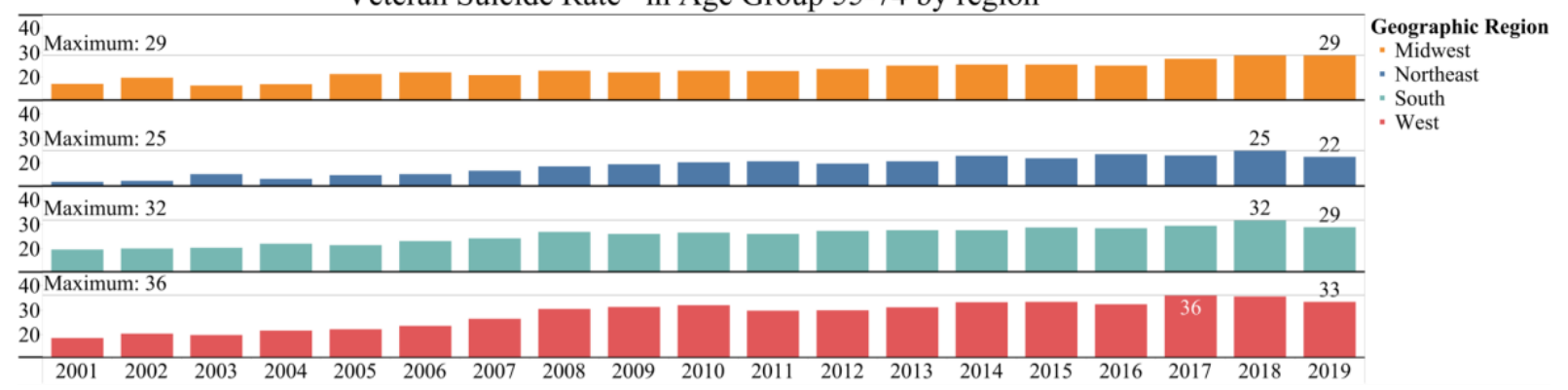


Veteran Suicide Rate* in Age Group 75+ by region

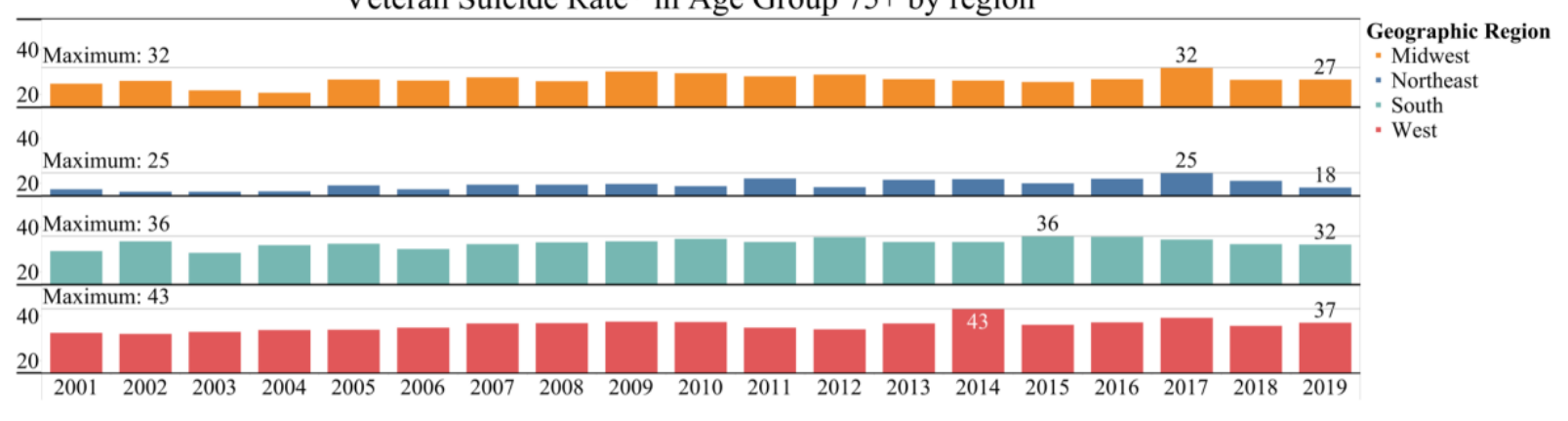

